\title{
Energy restriction and Roux-en-Y gastric bypass reduce postprandial $\alpha$-dicarbonyl stress in obese women with type 2 diabetes
}

\author{
Dionne E. Maessen ${ }^{1}$ • Nordin M. Hanssen ${ }^{1}$ • Mirjam A. Lips ${ }^{2}$ • Jean L. Scheijen ${ }^{1}$. \\ Ko Willems van Dijk ${ }^{2,3,4} \cdot$ Hanno Pijl $^{2,4}$ - Coen D. Stehouwer ${ }^{1}$. Casper G. Schalkwijk ${ }^{1}$
}

Received: 26 April 2016 / Accepted: 18 May 2016 / Published online: 16 June 2016

(C) The Author(s) 2016. This article is published with open access at Springerlink.com

\begin{abstract}
Aims/hypothesis Dicarbonyl compounds are formed as byproducts of glycolysis and are key mediators of diabetic complications. However, evidence of postprandial $\alpha$-dicarbonyl formation in humans is lacking, and interventions to reduce $\alpha$-dicarbonyls have not yet been investigated. Therefore, we investigated postprandial $\alpha$-dicarbonyl levels in obese women without and with type 2 diabetes. Furthermore, we evaluated whether a diet very low in energy (very low calorie diet [VLCD]) or Roux-en-Y gastric bypass (RYGB) reduces $\alpha$-dicarbonyl stress in obese women with type 2 diabetes.

Methods In lean $(n=12)$ and obese women without $(n=27)$ or with type 2 diabetes $(n=27)$, we measured the $\alpha$-dicarbonyls, methylglyoxal (MGO), glyoxal (GO) and 3-deoxyglucosone (3-DG), and glucose in fasting and postprandial plasma samples obtained during a mixed meal test. Obese women with type 2 diabetes underwent either a VLCD or RYGB.
\end{abstract}

Electronic supplementary material The online version of this article (doi:10.1007/s00125-016-4009-1) contains peer-reviewed but unedited supplementary material, which is available to authorised users.

Casper G. Schalkwijk

C.Schalkwijk@maastrichtuniversity.nl

1 Department of Internal Medicine, Laboratory for Metabolism and Vascular Medicine, Maastricht University Medical Center, Peter Debeyelaan 25, P.O. Box 5800, 6202

AZ Maastricht, the Netherlands

2 Department of Medicine, Division of Endocrinology, Leiden University Medical Center, Leiden, the Netherlands

3 Department of Human Genetics, Leiden University Medical Center, Leiden, the Netherlands

4 Einthoven Laboratory for Experimental Vascular Medicine, Leiden, the Netherlands
Three weeks after the intervention, individuals underwent a second mixed meal test.

Results Obese women with type 2 diabetes had higher fasting and particularly higher postprandial plasma $\alpha$-dicarbonyl levels, compared with those without diabetes. After three weeks of a VLCD, postprandial $\alpha$-dicarbonyl levels in diabetic women were significantly reduced (AUC MGO $-14 \%, \mathrm{GO}-16 \%$, 3-DG $-25 \%$ ), mainly through reduction of fasting plasma $\alpha$-dicarbonyls (MGO -13\%, GO -13\%, 3-DG -33\%). Similar results were found after RYGB.

Conclusions/interpretation This study shows that type 2 diabetes is characterised by increased fasting and postprandial plasma $\alpha$-dicarbonyl stress, which can be reduced by improving glucose metabolism through a VLCD or RYGB. These data highlight the potential to reduce reactive $\alpha$-dicarbonyls in obese individuals with type 2 diabetes.

Trial registration: ClinicalTrials.gov NCT01167959

Keywords Advanced glycation endproducts . $\alpha$-Dicarbonyls · Obesity · Type 2 diabetes .

Weight loss interventions

$\begin{array}{ll}\text { Abbreviations } \\ \text { 3-DG } & \text { 3-Deoxyglucosone } \\ \text { GB } & \text { Gastric banding } \\ \text { GO } & \text { Glyoxal } \\ \text { iAUC } & \text { Incremental area under the curve } \\ \text { MGO } & \text { Methylglyoxal } \\ \text { MMT } & \text { Mixed meal test } \\ \text { NGT } & \text { Normal glucose tolerance } \\ \text { Nrf2 } & \text { Nuclear factor (erythroid-derived 2)-like 2 } \\ \text { ROS } & \text { Reactive oxygen species } \\ \text { RYGB } & \text { Roux-en-Y gastric bypass } \\ \text { VLCD } & \text { Very low calorie diet }\end{array}$




\section{Introduction}

Postprandial glucose excursions are a detrimental factor in diabetic complications [1]. An important mechanism for postprandial glucose peaks contributing to increased risk of diabetic complications may be the formation of $\alpha$-dicarbonyls, which have been linked to a range of detrimental effects on cellular function [2].

We recently showed that individuals with type 2 diabetes had higher plasma levels of the $\alpha$-dicarbonyls methylglyoxal (MGO), glyoxal (GO) and 3-deoxyglucosone (3-DG), after a glucose load [3]. These highly reactive $\alpha$-dicarbonyls are mainly formed as glycolytic intermediates during glucose metabolism and rapidly interact with protein residues [2]. Importantly, $\alpha$-dicarbonyl stress has been linked to diabetic complications [2]. However, whether type 2 diabetes is associated with higher plasma $\alpha$-dicarbonyls after a meal is unknown.

Weight loss interventions, particularly energy restriction and bariatric surgery, have been linked to improved glucose metabolism and reduction of diabetic complications [4]. Interestingly, beneficial effects of energy restriction and bariatric surgery on insulin resistance seem to occur rapidly [5]. Thus, it is likely that $\alpha$-dicarbonyls will be rapidly reduced by these interventions.

Therefore, we investigated whether type 2 diabetes is associated with higher fasting and postprandial plasma $\alpha$-dicarbonyl levels compared with lean and obese women with normal glucose tolerance (NGT) and whether a diet very low in energy or Roux-en-Y gastric bypass (RYGB) reduces plasma $\alpha$-dicarbonyl levels in obese women with type 2 diabetes.

\section{Methods}

Study design and population The research design of this study has been described previously [5]. In short, we included obese women with NGT $(n=27)$ or type 2 diabetes $(n=27)$ who were eligible for dietary or surgical treatment. Agematched, healthy women with normal BMI $\left(<25 \mathrm{~kg} / \mathrm{m}^{2}\right.$; $n=12$ ) were included as controls for baseline comparisons. NGT obese individuals underwent either gastric banding (GB, $n=11)$ or RYGB $(n=16)$. Obese individuals with type 2 diabetes either underwent a diet very low in energy (very low calorie diet, VLCD, $n=12)$ or RYGB $(n=15)$. All women were characterised at baseline and obese women were additionally characterised 3 weeks after weight loss intervention. After discontinuing glucose-lowering medication for $48 \mathrm{~h}$ and overnight fasting $(\geq 10 \mathrm{~h}$ ), participants underwent a liquid mixed meal test (MMT) which consisted of $266 \mathrm{ml}$ (1673.6 kJ [400 kcal]) Nutridrink (Nutricia, Zoetermeer, the Netherlands) [5]. The study protocol (clinical trial registration no. NCT01167959) was approved by the medical ethical committee of the Leiden University Medical Center and all participants provided written informed consent. All analyses were performed blind.

Biochemical measurements $\alpha$-Dicarbonyl levels in EDTA plasma samples were measured as described previously [6]. Serum glucose levels and insulin resistance were assessed as described previously [5].

Calculation of area under the curve The AUC for $\alpha$-dicarbonyls and glucose measured during the MMT was
Table 1 Baseline characteristics of the study population

\begin{tabular}{llll}
\hline Characteristic & Lean $(n=12)$ & Obese NGT $(n=27)$ & Obese T2DM $(n=27)$ \\
\hline Age (years) & $49.2 \pm 6.2$ & $47.7 \pm 6.4$ & $51.0 \pm 7.1$ \\
Weight $(\mathrm{kg})$ & $64.4 \pm 7.2$ & $124.3 \pm 11.7^{* * *}$ & $117.2 \pm 17.1$ \\
BMI $\left(\mathrm{kg} / \mathrm{m}^{2}\right)$ & $21.7 \pm 1.6$ & $43.8 \pm 3.2^{* * *}$ & $42.0 \pm 5.5$ \\
Waist $(\mathrm{cm})$ & $78.0 \pm 6.0$ & $122.3 \pm 9.2^{* * *}$ & $123.2 \pm 11.0$ \\
Fat mass $(\%)$ & $35.5 \pm 2.4$ & $56.3 \pm 2.2^{* * *}$ & $55.7 \pm 4.4$ \\
$\mathrm{HbA}_{1 \mathrm{c}}(\mathrm{mmol} / \mathrm{mol})$ & $31.9 \pm 2.5$ & $36.1 \pm 7.8$ & $49.6 \pm 12.0^{\dagger \dagger \dagger}$ \\
$\mathrm{HbA}_{\mathrm{lc}}(\%)$ & $5.1 \pm 0.2$ & $5.5 \pm 0.7$ & $6.7 \pm 1.1^{\dagger \dagger}$ \\
Triacylglycerols $(\mathrm{mmol} / \mathrm{l})$ & $1.0 \pm 0.3$ & $1.4 \pm 0.6$ & $1.8 \pm 0.7^{\dagger}$ \\
NEFA (mmol/l) & $0.9 \pm 0.3$ & $1.0 \pm 0.4$ & $1.2 \pm 0.3$ \\
Total cholesterol $(\mathrm{mmol} / \mathrm{l})$ & $5.0 \pm 0.9$ & $4.6 \pm 1.0$ & $4.4 \pm 0.8$ \\
HDL cholesterol $(\mathrm{mmol} / \mathrm{l})$ & $1.7 \pm 0.3$ & $1.1 \pm 0.3^{* * *}$ & $1.1 \pm 0.3$ \\
LDL cholesterol $(\mathrm{mmol} / \mathrm{l})$ & $2.9 \pm 0.9$ & $2.9 \pm 0.9$ & $2.5 \pm 0.6$ \\
HOMA-IR & $0.3 \pm 0.1$ & $3.2 \pm 2.3^{* *}$ & $5.4 \pm 3.6^{\dagger}$ \\
\hline
\end{tabular}

Data are presented as means $\pm \mathrm{SD}$

Differences between the groups were tested using a one-way ANOVA with Bonferroni correction

$* * p<0.01$ and $* * * p<0.001$ for lean vs obese NGT individuals.

${ }^{\dagger} p<0.05$ and ${ }^{\dagger \dagger \dagger} p<0.001$ for obese NGT individuals vs obese individuals with type 2 diabetes (T2DM) 

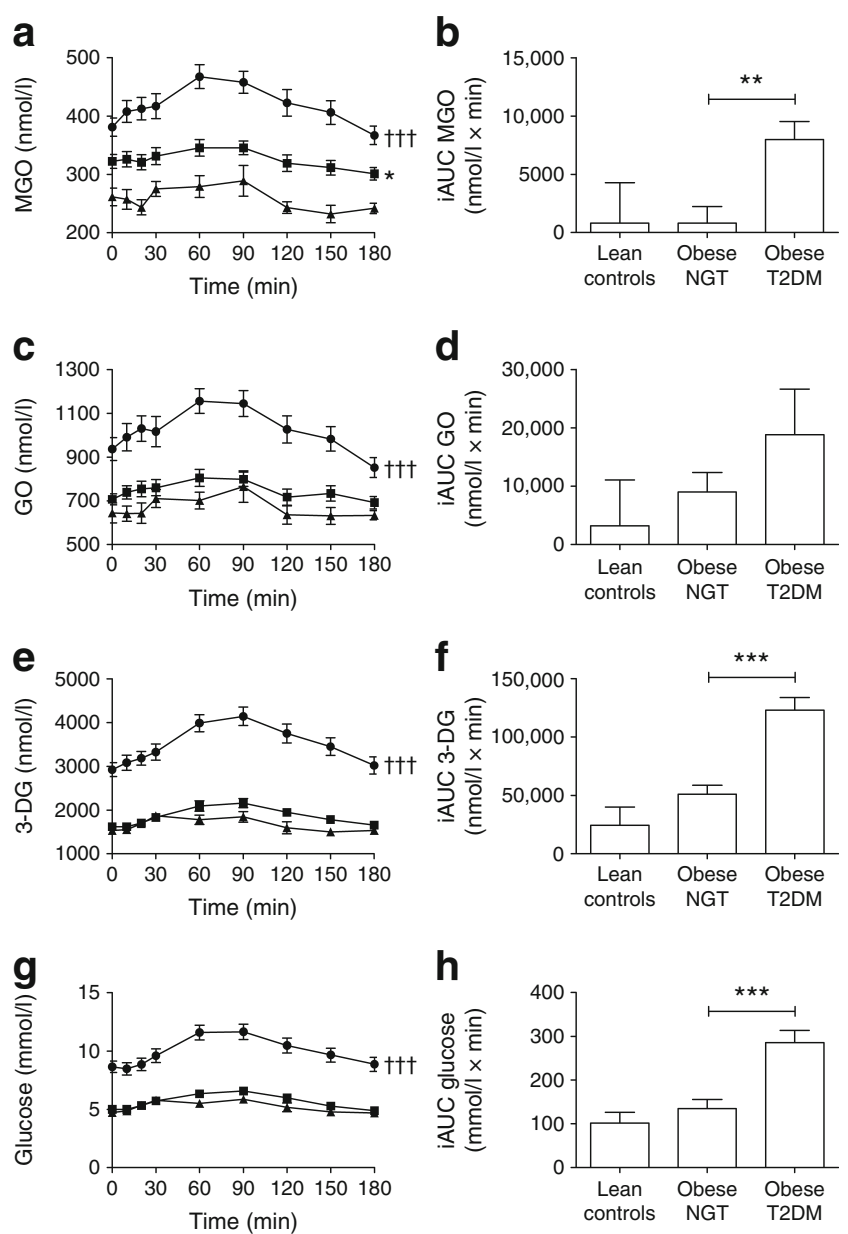

Fig. 1 Baseline plasma levels of $\alpha$-dicarbonyls and glucose before and during a mixed meal test. Plasma levels during the MMT of (a) MGO, (c) GO, (e) 3-DG and (g) glucose and iAUC, as calculated from the MMT of (b) MGO, (d) GO, (f) 3-DG and (h) glucose. Data are shown as means (SEM). Triangles, lean, $n=12$; squares, obese NGT, $n=27$; circles, obese type 2 diabetic individuals, $n=27$. Differences in postprandial curves during the mixed meal between the groups were tested with repeatedmeasures two-way ANOVA with Bonferroni correction. Differences in the iAUCs of MGO, GO, 3-DG and glucose between the groups were tested with one-way ANOVA with Bonferroni correction. ${ }^{*} p<0.05$, $* * p<0.01$ and $* * * p<0.001$ compared with lean individuals and ${ }^{+1} p<0.001$ compared with obese NGT individuals

calculated using the trapezoidal rule, where fasting levels were subtracted from each individual data point to specify postprandial excursions (incremental AUC, iAUC $[\mathrm{nmol} / 1 \times \min$ for $\alpha$-dicarbonyls and $\mathrm{mmol} / 1 \times \min$ for glucose]).

Statistical analysis Baseline levels of $\alpha$-dicarbonyls and glucose were compared using one-way ANOVA with Bonferroni correction. Two-way repeated measures ANOVA with Bonferroni correction was used to compare the three groups at baseline during the MMT. Adjustment for plasma glucose levels was performed with one-way ANCOVA with Bonferroni correction. Paired two-sided sample $t$ tests were used to compare $\alpha$-dicarbonyl and glucose levels before and after weight loss interventions. Data are expressed as means (SEM), unless otherwise stated. All statistical analyses were performed with IBM SPSS Statistics Software, version 20 (IBM Corporation, Armonk, NY, USA) and a two-sided $p$ value of $<0.05$ was considered statistically significant.

\section{Results}

Baseline characteristics of the study population are reported in Table 1 [5].

Fasting plasma MGO levels are higher in obese NGT women Fasting plasma MGO levels were higher in obese NGT individuals compared with lean individuals (see
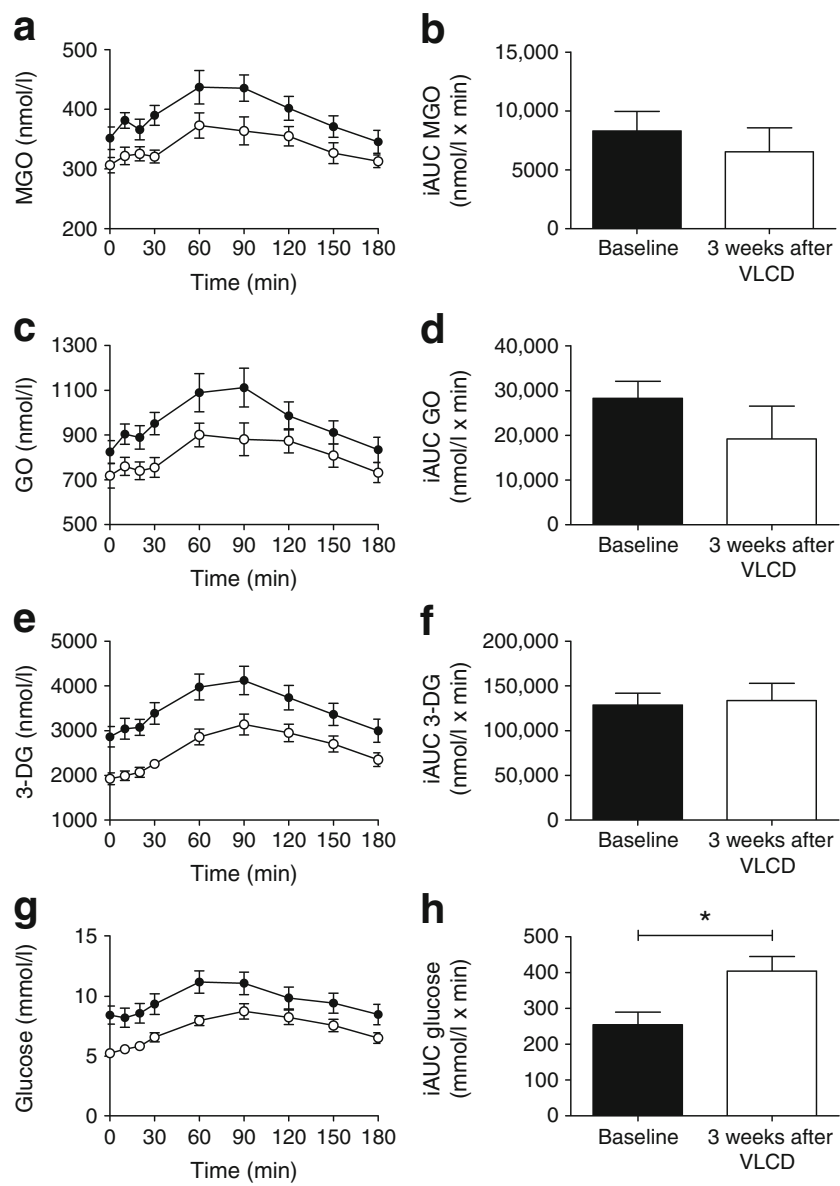

Fig. 2 Plasma levels of $\alpha$-dicarbonyls and glucose in obese individuals with type 2 diabetes after 3 weeks of a VLCD. Plasma levels during the MMT of (a) MGO, (c) GO, (e) 3-DG and (g) glucose and iAUC, as calculated from the MMT, of (b) MGO, (d) GO, (f) 3-DG and (h) glucose. Data are shown as means (SEM). Black circles, obese type 2 diabetes individuals before the VLCD; open circles, obese type 2 diabetes individuals 3 weeks after the VLCD, $n=12$. Differences in fasting levels and the iAUCs of MGO, GO, 3-DG and glucose were tested with paired two-sided samples $t$ tests. ${ }^{*} p<0.05$ compared with baseline 
electronic supplementary material [ESM] Table 1, $p<0.05$ ), which remained significant after adjustment for glucose (ESM Table 1). None of the other fasting $\alpha$-dicarbonyls were increased in obese NGT individuals (ESM Table 1). Furthermore, iAUCs of all plasma $\alpha$-dicarbonyls and glucose did not differ between lean and obese NGT individuals (Fig. 1b,d,f,h).

Fasting and postprandial $\alpha$-dicarbonyl stress is higher in obese women with type 2 diabetes When we compared obese individuals with NGT and those with type 2 diabetes at baseline, we found significantly higher fasting plasma levels of $\alpha$-dicarbonyls (ESM Table 1, $p<0.01$ for all) in those with type 2 diabetes. Particularly, the postprandial iAUCs of MGO, 3-DG and glucose were higher $(p<0.01$ for all in obese individuals with type 2 diabetes compared with obese NGT individuals (Fig. 1b,f,h). We observed a similar, yet non-significant difference between obese NGT and type 2 diabetic individuals for the iAUC of GO (Fig. 1d). After adjustment for glucose, all significant differences between obese individuals without and with type 2 diabetes were attenuated (ESM Table 1).

Fasting plasma MGO levels are reduced by RYGB in obese NGT women within 3 weeks In obese NGT individuals, we observed reduced fasting MGO levels after RYGB $(p<0.05)$, but no significant reduction in other $\alpha$-dicarbonyls (ESM Fig. 1). Weight loss through GB in obese NGT individuals did not lead to changes in $\alpha$-dicarbonyl stress (ESM Fig. 2).

A VLCD and RYGB reduce fasting and postprandial plasma $\alpha$-dicarbonyl stress in obese women with type 2 diabetes In obese type 2 diabetic individuals, postprandial $\alpha$-dicarbonyls and glucose levels were decreased after 3 weeks of a VLCD (Fig. 2a,c,e,g). Indeed, the total load of $\alpha$-dicarbonyls and glucose after a meal, as reflected by the AUC, was substantially decreased by the VLCD (data not shown, $p<0.05$ for all). This effect was mostly attributable to decreased fasting $\alpha$-dicarbonyls (MGO and 3-DG, $p<0.05$ for both), as the postprandial $\alpha$-dicarbonyl excursions (iAUC) after a meal were similar before and after the VLCD (Fig. 2b,d,f). Fasting plasma GO levels did not change significantly after 3 weeks of the VLCD, but a non-significant $(p=0.11)$ reduction was observed. Comparable findings regarding the effect on postprandial $\alpha$-dicarbonyl stress were found 3 weeks after RYGB (ESM Fig. 3).

\section{Discussion}

This study shows that obese individuals with type 2 diabetes have higher fasting and particularly higher postprandial plasma $\alpha$-dicarbonyl levels than individuals without diabetes. Moreover, VLCD and RYGB reduced plasma $\alpha$-dicarbonyl stress in obese type 2 diabetic individuals, mainly through reduction of fasting plasma $\alpha$-dicarbonyls.

In obese NGT individuals, we found higher fasting plasma MGO levels compared with lean individuals, but we observed no further postprandial increase. Interestingly, these increased fasting MGO levels were reduced by RYGB. The elevated fasting MGO levels in obese individuals compared with healthy individuals remained significant after adjustment for glucose. Therefore, other pathways may contribute to increased MGO formation in obese NGT individuals. In contrast, fasting and postprandial plasma levels of the other $\alpha$-dicarbonyls were not affected by the presence of obesity.

Our current findings on increased postprandial $\alpha$-dicarbonyl stress in type 2 diabetes, together with similar findings in type 1 diabetes [7], are in line with our previous work, in which we demonstrated increased $\alpha$-dicarbonyl stress in type 2 diabetic individuals after an oral glucose load [3]. Therefore, it is likely that the increased $\alpha$ dicarbonyl levels after a meal result from increased postprandial glucose levels. Furthermore, the possible role of other substrates for $\alpha$-dicarbonyl formation, such as reduced lipid oxidation and reactive oxygen species (ROS) cannot be excluded. ROS are known to increase postprandially in type 2 diabetes [8] and, therefore, reduction of ROS by weight loss could also be responsible for lower postprandial MGO stress, but this phenomenon deserves further investigation. Additionally, a VLCD may reduce MGO via induction of nuclear factor (erythroid-derived 2)-like 2 (Nrf2), an inducer of the MGO-detoxifying enzyme glyoxalase-1 [9].

Our current findings are of potential clinical importance, since $\alpha$-dicarbonyls have been identified as potential key mediators of diabetic complications. Of the three major $\alpha$-dicarbonyls, MGO is the most potent glycating agent [2]. MGO seems to play a crucial role in the development of diabetic complications, particularly via induction of endothelial dysfunction. Therefore, excursions in MGO at the fasting and postprandial level in type 2 diabetic individuals are a potential target for prevention and treatment of diabetic complications. For example, quenching $\alpha$-dicarbonyls may be possible with the vitamin $\mathrm{B}_{6}$ analogue, pyridoxamine [10]. Furthermore, MGO may be reduced via induction of glyoxalase-1, using inducers of $\mathrm{Nrf} 2$ [9].

This is the first study that describes increased postprandial $\alpha$-dicarbonyl stress in obese type 2 diabetic individuals, and its reduction by weight loss. Future work should investigate whether a VLCD and RYBG achieve health improvements and lower cardiovascular risk through reduced $\alpha$-dicarbonyl stress. A limitation of this study was that we could not include 
a control group that remained weight-stable. Nevertheless, we report on two distinct interventions that aimed to induce weight loss, showing comparable results. Another limitation was that we lacked statistical power to investigate mediating factors through which $\alpha$-dicarbonyl levels were improved by VLCD and RYGB. Additionally, we investigated women only in the current study, but we have no basis to assume that our results would be different in men. Furthermore, our study does not reveal which tissues contribute to the plasma pool of $\alpha$-dicarbonyls. Although we hypothesise that plasma $\alpha$-dicarbonyls are derived from insulin-independent cells which are in close contact with blood, such as endothelial cells and erythrocytes, animal studies are needed to fully address this issue.

In conclusion, we demonstrated increased postprandial $\alpha$-dicarbonyl stress in obese individuals with type 2 diabetes, which can be reduced by a VLCD or RYGB. These data highlight the potential to reduce $\alpha$-dicarbonyls as a target to prevent or delay development of complications in obese type 2 diabetic individuals.

Funding This study was performed within the framework of the Center for Translational Molecular Medicine (www.ctmm.nl); Prediction and Early Diagnosis of Diabetes and Diabetes-related Cardiovascular Complications (PREDICCt) project (grant 01 $\mathrm{C}$-104). The study was performed with an unrestricted grant from the Dutch Obesity Clinic. This study was supported by the Centre for Medical Systems Biology (CMSB) and the Netherlands Consortium for Systems Biology (NCSB), both within the framework of the Netherlands Genomics Initiative (NGI)/Netherlands Organization for Scientific Research (NWO).

Duality of interest statement The authors declare that there is no duality of interest associated with this manuscript.

Contribution statement All authors contributed substantially to the design of the study or experimental procedures. DEM and NMH analysed and interpreted data. JLS performed laboratory analyses. DEM drafted the manuscript. All authors critically revised the manuscript and approved this version to be published. CGS is the guarantor of this work.
Open Access This article is distributed under the terms of the Creative Commons Attribution 4.0 International License (http:// creativecommons.org/licenses/by/4.0/), which permits unrestricted use, distribution, and reproduction in any medium, provided you give appropriate credit to the original author(s) and the source, provide a link to the Creative Commons license, and indicate if changes were made.

\section{References}

1. Madsbad S (2016) Impact of postprandial glucose control on diabetes-related complications: how is the evidence evolving? J Diabet Complicat 30:374-385

2. Maessen DE, Stehouwer CD, Schalkwijk CG (2015) The role of methylglyoxal and the glyoxalase system in diabetes and other age-related diseases. Clin Sci (Lond) 128:839-861

3. Maessen DE, Hanssen NM, Scheijen JL et al (2015) Post-glucose load plasma alpha-dicarbonyl concentrations are increased in individuals with impaired glucose metabolism and type 2 diabetes: the CODAM study. Diabetes Care 38:913-920

4. Schauer PR, Bhatt DL, Kirwan JP et al (2014) Bariatric surgery versus intensive medical therapy for diabetes-3-year outcomes. N Engl J Med 370:2002-2013

5. Lips MA, de Groot GH, van Klinken JB et al (2014) Calorie restriction is a major determinant of the short-term metabolic effects of gastric bypass surgery in obese type 2 diabetic patients. Clin Endocrinol (Oxf) 80:834-842

6. Scheijen JL, Schalkwijk CG (2014) Quantification of glyoxal, methylglyoxal and 3-deoxyglucosone in blood and plasma by ultra performance liquid chromatography tandem mass spectrometry: evaluation of blood specimen. Clin Chem Lab Med 52:85-91

7. Beisswenger PJ, Howell SK, O'Dell RM, Wood ME, Touchette AD, Szwergold BS (2001) alpha-Dicarbonyls increase in the postprandial period and reflect the degree of hyperglycemia. Diabetes Care 24:726-732

8. Ceriello A, Bortolotti N, Motz E et al (1999) Meal-induced oxidative stress and low-density lipoprotein oxidation in diabetes: the possible role of hyperglycemia. Metabolism 48:1503-1508

9. Xue M, Rabbani N, Momiji H et al (2012) Transcriptional control of glyoxalase 1 by $\mathrm{Nrf} 2$ provides a stress-responsive defence against dicarbonyl glycation. Biochem J 443:213-222

10. Maessen DE, Brouwers O, Gaens KH et al (2016) Delayed intervention with pyridoxamine improves metabolic function and prevents adipose tissue inflammation and insulin resistance in high-fat diet-induced obese mice. Diabetes 65:956-966 